$\begin{array}{cl}\text { PRAMANA } & \text { (C) Indian Academy of Sciences } \\ \begin{array}{c}\text { journal of } \\ \text { physics }\end{array} & \begin{array}{l}\text { Vol. } 65, \text { No. } 5 \\ \text { November } 2005 \\ \text { pp. } 777-786\end{array}\end{array}$

\title{
Optically-driven red blood cell rotor in linearly polarized laser tweezers
}

\author{
MANAS KHAN ${ }^{1}$, SAMARENDRA K MOHANTY ${ }^{1,3}$ and A K SOOD ${ }^{1,2}$ \\ ${ }^{1}$ Department of Physics, Indian Institute of Science, Bangalore 560 012, India \\ ${ }^{2}$ Unit on Nanoscience and Technology-DST, Indian Institute of Science, \\ Bangalore 560 012, India \\ ${ }^{3}$ Present address: Biomedical Applications Section, Centre for Advanced Technology, \\ Indore 452 013, India \\ E-mail: manas@physics.iisc.ernet.in; asood@physics.iisc.ernet.in
}

\begin{abstract}
We have constructed a dual trap optical tweezers set-up around an inverted microscope where both the traps can be independently controlled and manipulated in all the three dimensions. Here we report our observations on rotation of red blood cells (RBCs) in a linearly polarized optical trap. Red blood cells deform and become twisted in hypertonic phosphate buffer saline and when trapped, experience an unbalanced radiation pressure force. The torque generated from the unbalanced force causes the trapped RBC to rotate. Addition of $\mathrm{Ca}^{++}$ions in the solution, keeping the osmolarity same, makes the cell membranes stiffer and the cells deform less. Thus the speed of rotation of the red blood cells can be controlled, as less deformation and in turn less asymmetry in shape produces less torque under the radiation pressure resulting in slower rotation at the same laser power.
\end{abstract}

Keywords. Rotation of red blood cell; optical tweezers, dual optical trap.

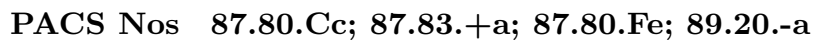

\section{Introduction}

The application of optical tweezers in trapping and manipulating single cells [1] has opened new prospects in basic research related to life science, starting from single cell molecular biology [2] to laser-assisted in-vitro fertilization [3], covering a diverse field of applications including development of cell biosensors and cellular microchips [4,5]. Different mechanical aspects of different cells and even single DNA molecules [6] are investigated by the controlled light force. Human erythrocytes, being one of the prime interests in drug therapy, are well investigated in single cell level with the help of optical trap. Rotations of erythrocytes in different conditions and environments have been observed and reported [7-11]. These recent results on rotations of red blood cells [7-11] inspired us to readdress the issue as the reported results are not in complete agreement with each other. It was reported $[8,10]$ that in hypertonic buffer solutions RBCs take a meniscus shape and rotate 


\section{Manas Khan, Samarendra K Mohanty and A K Sood}

when trapped. The speed of rotation is less in the case of malaria infected red blood cells. In contrast, it was argued $[7,9]$ that the red blood cells get folded when they are trapped and become birefringent. Those folded cells having birefringence property rotate only in circularly polarized light. Dharmadhikari et al [7] have also reported the rotation of malaria infected RBCs under linearly polarized optical trap. We have done a systematic study on normal RBCs and tried to investigate the effect of cell membrane rigidity on the rotation by adding bivalent ions in the phosphate buffer saline. We have observed the normal red blood cells to rotate under linearly polarized optical trap but only when they are in hypertonic phosphate buffer saline. In hypertonic osmolar condition, the fluid comes out from the cells and the cells become thin and twisted. The twisted asymmetric structures get vertically aligned when trapped and start rotating. We present a simple model to explain our observations. When in hypertonic osmolar solution, because of the asymmetry in shape, the trapped erythrocyte experiences unbalanced scattering forces and hence a net torque. The RBC rotates under the radiation pressure by virtue of its shape asymmetry that does not have a mirror plane of symmetry passing through the optic axis. Our model predicts that this rotation mechanism is independent of the state of polarization of the trapping beam and solely depends on shape asymmetry [12]. Since orientation and rotation of flat particles by rotating the plane of polarization of the linearly polarized trapping beam have been observed [13], it might be tempting to infer that in isotonic buffer solution the disk-like RBCs will rotate under the circularly polarized optical trap. However this speculation is not right as it has been shown experimentally that the speed of rotation of the plane of polarization in the case of circularly polarized light, being equal to the frequency of light, is too fast for a micrometer sized object to follow [13]. So there is no additional effect of circularly polarized light on RBCs in isotonic buffer solution and all our observations are expected to be the same even under the circularly polarized trapping beam. In addition to this we have also noticed that the rate of rotation can be controlled by manipulating the degree of deformation of the erythrocytes. Addition of $\mathrm{Ca}^{++}$ions stiffens the cell membrane and therefore at the same osmolarity level of the solution, the cells deform less as the concentration of $\mathrm{Ca}^{++}$ions is increased. The less asymmetric structure experiences lower torque resulting in slower rotation. Here we report our experiments and results of the red blood cell rotations along with a detailed discussion of our dual optical trap set-up and its calibration.

\section{The set-up}

\subsection{The design}

We have constructed a dual optical trap set-up around an inverted microscope. Figure 1 is a schematic of the optical layout. The set-up is built on an active vibration isolation system to isolate the noise due to vibrations. A linearly polarized CW laser beam coming out from a $2.5 \mathrm{~W} 1064 \mathrm{~nm}$ diode pumped solid state $\mathrm{Nd}: \mathrm{YVO}_{4}$ laser (model T20-V-106C-2500; Spectra Physics Inc., USA) is split up into two beams and are used for two independent optical traps. A beam isolator 


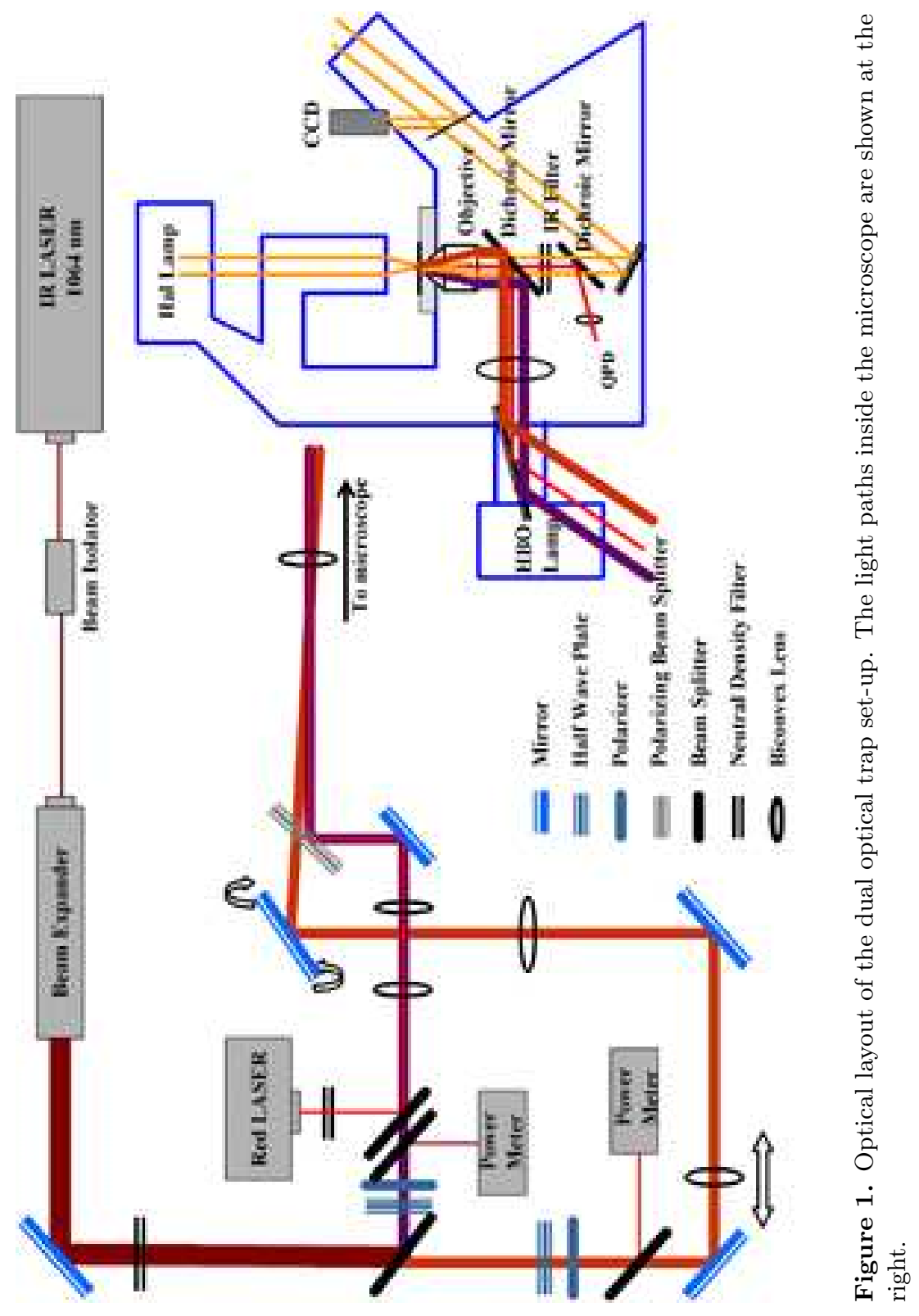




\section{Manas Khan, Samarendra K Mohanty and A K Sood}

(model IO-3-YAG-HP; Optics for Research, USA) is used just after the laser head to block the back reflection of the laser beam from different optics. The laser beam is expanded by a beam expander (model ELQ-51-40X-YAG; Optics for Research, USA) before splitting up into two beams using a 70:30 plate beam splitter (model 03 BFT 027; Melles Griot, The Netherlands). The plane of polarization and the intensity of both the beams can be controlled independently by using a half-wave plate (model 02 WRQ 031/1064; Melles Griot, The Netherlands) and then a polarizer (model 16 PPB 195; Melles Griot, The Netherlands) in the beam path. Both the half-wave plate and the polarizer are fixed on rotatable mounts and thus can be rotated freely about the optic axis. A rotation of the half-wave plate by an angle $\theta$ with respect to the pass axis of the polarizer results in a change in the intensity by a factor $\left(\cos ^{2} 2 \theta\right)$. Using the half-wave plate, the plane of polarizations of the two beams are made perpendicular to each other and hence they can be finally merged using a polarizing beam splitter cube (Melles Griot, The Netherlands) without any intensity loss. The merged beams are sent through the epifluorescence port of an inverted microscope (model Axiovert 200; Carl Zeiss, Germany) and are focused tightly by a 1.4 numerical aperture $100 \times$ objective. A slight angle between the direction of the beams makes them focus at different spatial points at the sample plane. To make both the traps independently steerable, we introduce a piezo-controlled (model K-110-00; Piezosystem Jena GmbH, Germany) mirror with nano- $\theta_{x} \theta_{y}$ tilt in the path of the second (less intense) beam. By controlling the mirror tilt, the final X,Y-position of the second trap at the sample plane can be manipulated. A piezo-controlled XYZ nanostage (model P-517.3CL; Physik Instrumente, Germany) is attached on the microscope stage to move the sample with respect to the trap positions. Thus the first trap can be moved three dimensionally with respect to the sample only by controlling the XYZ piezo-stage even though the first beam is not steered by any $\theta_{x} \theta_{y}$ tilting mirror. At the time of steering the second beam with the $\theta_{x} \theta_{y}$ tilting mirror, the following conditions should be fulfilled: (i) the beam should pivot around the entrance aperture of the microscope objective and (ii) the beam should retain the same degree of 'overfilling' of the objective entrance aperture. Both of these requirements can be fulfilled if the $\theta_{x} \theta_{y}$ tilting mirror is placed at the conjugate image plane of the entrance aperture of the microscope objective. We place a biconvex lens, that along with the lenses inside the microscope make a telescopic arrangement, in the beam path to the epifluorescence port to image the objective back aperture on the tilting mirror. For the independent Z-movement of each trap, we insert another telescopic pair of biconvex lenses in each of the beam paths. By moving one of the lenses slightly along the optic axis of the beam, the convergence of each beam can be controlled and hence the Z-position of both the traps can be manipulated at the sample plane. A $660 \mathrm{~nm}$ red laser (model ML101J8 with TCLDM9/M; Thorlabs Inc., USA) beam is merged with the first infrared beam to track the position of the trapped particle. The two infrared beams and the red beam enter the microscope through the epifluorescence port and are reflected to the objective by a dichroic mirror (Chroma Technology Corp., USA). The dichroic mirror transmits the halogen illumination of the microscope coming through the sample, making the visualization of the sample possible. It passes $50 \%$ of the the back-scattered red light but reflects the infrared beams completely. The back-scattered red light is reflected by another dichroic mirror, mounted inside the 


\section{Optically-driven $R B C$ rotor}

microscope, before falling on the quadrant photo diode (QPD) that detects the deflection of the back-scattered beam and in turn the position fluctuations of the trapped particle. The experiments are recorded on computer hard-disk by an image grabber card (model PCI-1428; National Instruments, USA) connected to a CCD camera (model UP-1830-CL; Uniq Vision Inc., USA) attached at the binocular imaging port of the microscope.

Here we present our recent experiments on erythrocytes using only a single trap.

\subsection{Calibration of the set-up}

A dielectric particle at the point of focus of the laser beam experiences a gradient force towards the region of highest intensity and a scattering force in the direction of the incident beam. For high intensity gradients, the gradient force dominates and causes a restoring force that defines the potential landscape of the trap. A particle would be very strongly trapped if the potential well is steeper. The variation of the trap stiffness with the laser power gives the efficiency of the set-up. There are many methods available for the calibration [14] of an optical trap. For small displacements $( \pm 250 \mathrm{~nm}$ for $1 \mu \mathrm{m}$ diameter bead), the restoring force is, to a good approximation, harmonic and can be given by $F=-\kappa x$, where $F$ is the restoring force, $\kappa$ is the force constant and $x$ is the displacement of the bead.

Here we discuss some typical calibration results of our set-up for a $2 \mu \mathrm{m}$ diameter polystyrene bead. The calibration was done using equipartition theorem method. It is known from the equipartition theorem that for a particle confined in a threedimensional potential well, in every direction,

$$
\frac{1}{2} k_{\mathrm{B}} T=\frac{1}{2} \kappa_{i}\left\langle x_{i}^{2}\right\rangle \Rightarrow \kappa_{i}=\frac{k_{\mathrm{B}} T}{\left\langle x_{i}^{2}\right\rangle}, \quad i=1,2,3 .
$$

We measured $\kappa_{1}$ and $\kappa_{2}$ at different laser powers $(P)$ and calculated $\mathrm{d} \kappa / \mathrm{d} P$, the efficiency of the set-up. The trapped bead was imaged at 30 frames per second rate and the distribution of the bead position was evaluated from the images using a LabVIEW VI and plotted. The Gaussian fit to the bar-plot gives the mean square displacement, $\left\langle x_{1}^{2}\right\rangle$, which in turn gives the trap stiffness $\kappa_{1}$, following eq. (1).

Figure $2 \mathrm{a}$ shows the position distribution of a $2 \mu \mathrm{m}$ bead along the X-direction at a laser power $P=27.8 \mathrm{~mW}$. The $x$-axis of the bar-plot is represented in terms of image pixels. The black curve is the Gaussian fit to the bar-plot. In figure $2 \mathrm{~b}$ a plot of trap stiffness $\left(\kappa_{1}\right)$ against laser power is shown. The trap stiffness increases linearly with increasing laser power with a slope of $\mathrm{d} \kappa / \mathrm{d} P=1.09 \mathrm{pN} /(\mu \mathrm{m} \cdot \mathrm{mW})$.

\section{Experiments}

Normal red blood cells were extracted from fresh human blood (treated with the anticoagulant, $\mathrm{C}_{10} \mathrm{H}_{14} \mathrm{~N}_{2} \mathrm{Na}_{2} \mathrm{O}_{8} \cdot 2 \mathrm{H}_{2} \mathrm{O}$ ). The cells were taken in isotonic, hypotonic and hypertonic phosphate buffer solutions with osmolarities $150 \mathrm{mOsm} / \mathrm{l}, 300$ $\mathrm{mOsm} / \mathrm{l}$ and $1200 \mathrm{mOsm} / \mathrm{l}$ respectively. The solutions were prepared adding the required amount of $\mathrm{NaCl}$ in the phosphate buffer solution. The cells were treated 

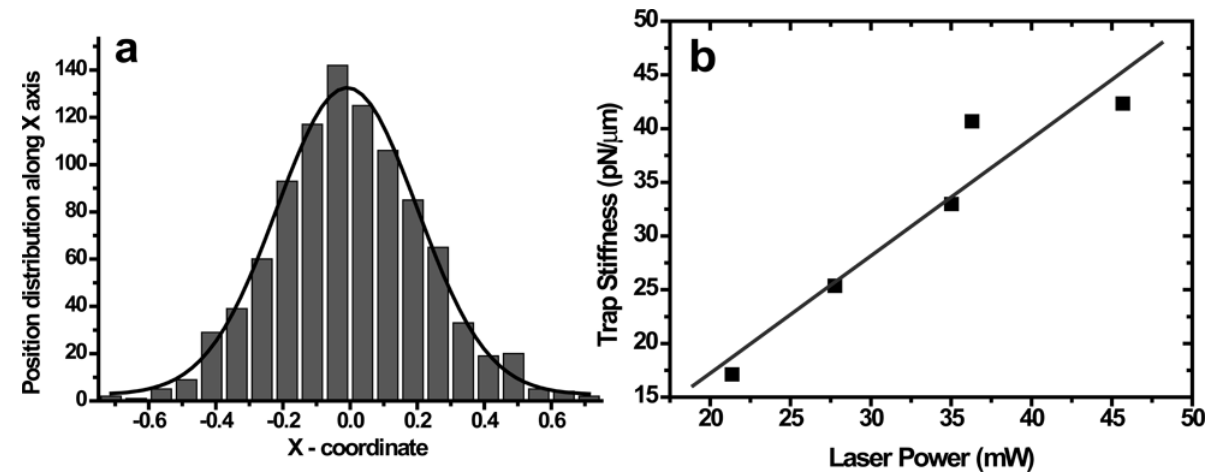

Figure 2. The bar-plot (a) shows the position distribution of a $2 \mu \mathrm{m}$ polystyrene bead along the X-direction at laser power $P=27.8 \mathrm{~mW}$. The $x$-axis of the bar-plot is represented in terms of image pixels. The black curve is the Gaussian fit to the bar-plot. (b) A plot of trap stiffness $\left(\kappa_{1}\right)$ against laser power is shown.

in the buffer solutions for nearly two hours and then trapped, one at a time, using a linearly polarized infrared $(1064 \mathrm{~nm})$ laser beam. We repeated the experiments at different laser powers with red blood cells in all three osmolar conditions. In each set of observation, more than twenty cells were trapped and observed separately.

Then we prepared three different hypertonic solutions, all with osmolarity 1200 $\mathrm{mOsm} / \mathrm{l}$ but having different concentrations of $\mathrm{Ca}^{++}$ions. We used hypertonic phosphate buffers with ion concentration ratios $\mathrm{Na}^{+}: \mathrm{Ca}^{++}=6: 1, \mathrm{Na}^{+}: \mathrm{Ca}^{++}=2: 1$ and with only $\mathrm{Ca}^{++}$ions in the solution. Red blood cells were kept in each solution separately for more than two hours before trapping with the same linearly polarized infrared beam. The experiments were recorded at 30 frames per second with a CCD camera attached with an inverted microscope. The rotation speeds of the red blood cells in different media and at different laser powers were calculated from the recorded image frames.

\section{Results}

\subsection{Observations}

The red blood cells deformed and became twisted in the hypertonic osmolar condition (without any $\mathrm{Ca}^{++}$ions). When they were in horizontal orientation, disfigured circular profiles were observed, but as the orientation changed randomly, the projection also changed. In the vertical orientation they looked like elongated asymmetric structures. After switching on the trap, the cells come to the trap and necessarily become vertical to optimize the interaction energy with the optical trap. Switching off the trap brought them back to the randomly oriented state. All of the cells rotated spontaneously in the linearly polarized optical trap. We did not observe any noticeable change in shape, like folding of the red blood cells when they were trapped. We varied the laser power from $10 \mathrm{~mW}$ to $60 \mathrm{~mW}$ and a linear increase 


\section{Optically-driven $R B C$ rotor}

in rotational speed was observed. As a typical value, at $50 \mathrm{~mW}$ of laser power the cells were observed to rotate at $180 \mathrm{rpm}$. The RBCs in isotonic solution had normal biconcave shapes and they got aligned vertically in the trap without any change in their shape. None of those symmetric shaped cells showed any rotation even at the highest laser power. In hypotonic medium, the cells swelled up and became almost spherical. The trapped one and the free cells looked alike. They did not show any deformation or rotation under the optical trap.

The results of treating the cells with hypertonic solutions containing different concentrations of $\mathrm{Ca}^{++}$ions were more dramatic. Bivalent ions were added in the solution to increase the rigidity of the cell membranes. As the concentration of $\mathrm{Ca}^{++}$ions increased, the cells deformed less even though the osmolarities were same for all the solutions. At the highest concentration of calcium ions, the cells retained their normal shape but their membranes (topology) became very rough. With increasing concentration of $\mathrm{Ca}^{++}$ions, the deformations and also the rotational speeds of the red blood cells were observed to decrease significantly. The number fraction of the cells that showed rotation under optical trap also decreased with increasing concentration of the calcium ions. The cells in the hypertonic solution with only bivalent ions did not show any rotation even at the highest laser power.

Figure 3 shows the time sequences of the RBC rotors at the same laser power but in different osmolar conditions. The orientation of each cell has been denoted by an arrow. In panel A, the RBC is in hypertonic buffer solution with osmolarity $1200 \mathrm{mOsm} / \mathrm{l}$ without any bivalent ions. The speed of rotation is the highest (at the same laser power of $50 \mathrm{~mW}$ ) in this case compared to panels $\mathrm{C}$ and $\mathrm{D}$. In the second row (B), two RBCs are shown. The one at the center is in the trap and shows rotation whereas the other is a free $\mathrm{RBC}$. The free $\mathrm{RBC}$ is also in vertical orientation and hence looks similar to the trapped RBC. Here the medium is the same as that of panel A. In panel $\mathrm{C}$, the cells are in hypertonic buffer solution with $\mathrm{Na}^{+}: \mathrm{Ca}^{++}=6: 1$, with the same osmolarity, $1200 \mathrm{mOsm} / \mathrm{l}$, and a significant change in the rotational speed is observed. In the last panel $\mathrm{D}$, the cells are in a hypertonic solution with only calcium salt. Though the osmolarity of the solution is the same, the behavior is completely different. In this case the RBC does not rotate at all. The deformation of the cell is less but the membrane becomes rough and rigid.

\subsection{Theoretical understanding}

The shapes of human erythrocytes in different osmolar conditions are well investigated $[15,16]$. In the isotonic solution the normal erythrocytes are biconcave disk-like structures with diameter $\sim 6 \mu \mathrm{m}$. In hypertonic solutions the cytoskeleton fluid comes out from the cells and the cells become thin. At very high osmolarity ( 1200 mOsm/l), the cells deform and bear twisted disk-like structures. In hypotonic solution, the cells get swollen and become almost spherical.

When a few drops of the RBC sample is observed under the microscope, the cells would be visible with all possible orientations. In hypotonic solution, as they have nearly spherical shapes, all the cells look alike even in different orientations. The biconcave shapes are prominently visible when the cells are in isotonic solution. In 
$\mathbf{A}$

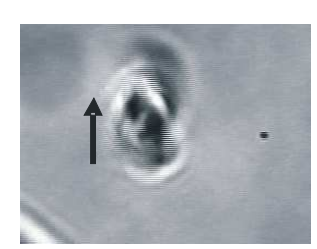

$\mathbf{B}$

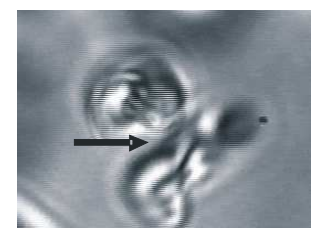

C

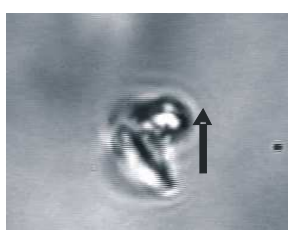

D

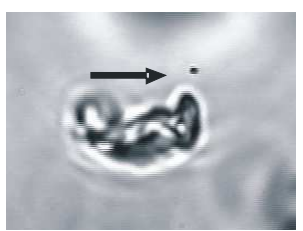

$\mathbf{t}=0.06 \mathrm{sec}$
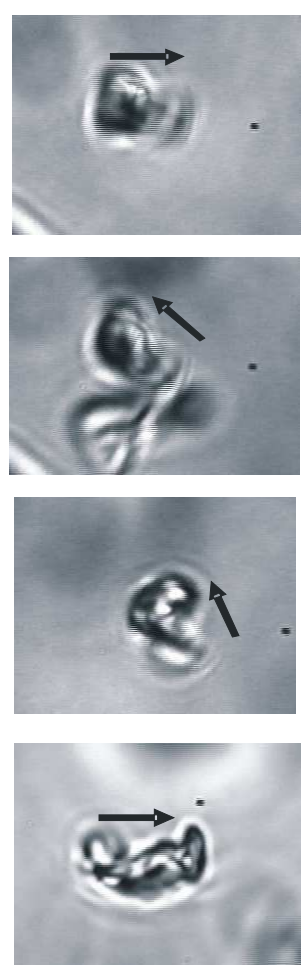

$\mathbf{t}=\mathbf{0 . 1 3} \mathrm{sec}$
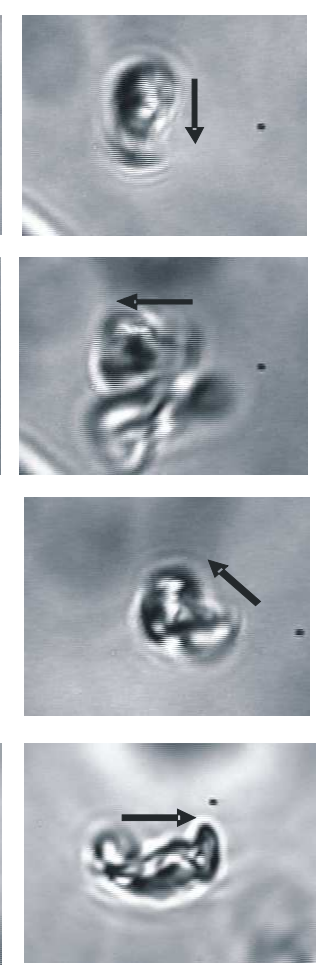

Figure 3. Time sequences of four RBC rotors in different osmolar conditions but at the same laser power $(50 \mathrm{~mW})$ are shown. In all the cases the osmolarities of the solutions are $1200 \mathrm{mOsm} / \mathrm{l}$. For the first two rows (A, B), the solutions contain only sodium salt. In the second row (B), two red blood cells are shown in each frame. The RBC at the center is in optical trap and shows rotation. The third row $(\mathrm{C})$ shows the rotation of a $\mathrm{RBC}$ in hypertonic buffer with $\mathrm{Na}^{+}: \mathrm{Ca}^{++}=6: 1$. The fourth row (D) shows time sequences of a $\mathrm{RBC}$ in calcium salt solution with same osmolarity, $1200 \mathrm{mOsm} / \mathrm{l}$. In this case the $\mathrm{RBC}$ does not show any rotation. The magnification factor for all the images are same.

vertical orientation, the cells appear oblate as only the side projections are visible. The circular faces are observed as soon as they become horizontal. In a similar fashion, in hypertonic solution, the thin twisted disk-like cells show their deformed circular face only when they are horizontal. The vertically oriented cells will show only their side projections that are more like asymmetric elongated wrapped bodies. The RBCs that are under the optical trap, necessarily become vertically aligned to optimize the interaction energy (with the optical trap) $W$, where $W \propto \int I \mathrm{~d} v$.

Now to understand the rotation mechanism of the RBCs in hypertonic solution, we take a simple model shape (as depicted in figure 4) imitating the deformed erythrocyte shapes in hypertonic buffer solution. If the wrapped disk-like structure does not have a plane of mirror symmetry passing through the optic axis, the 


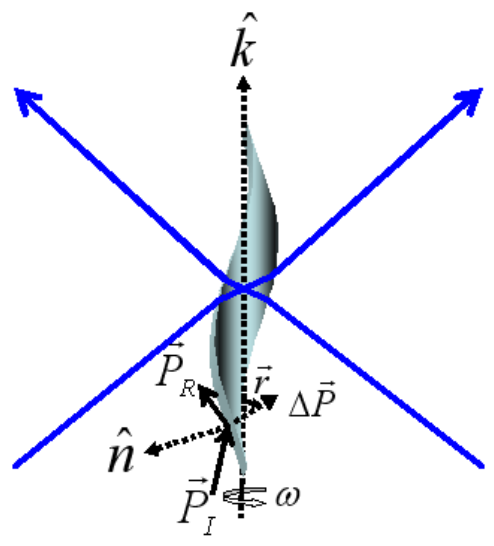

Figure 4. A model shape, representing a deformed erythrocyte in hypertonic buffer solution, is shown. The momentum change of the incident ray causes an unbalanced and off-centered force which contribute to a total nonzero torque enabling a trapped asymmetric erythrocyte to rotate about the optic axis.

asymmetry in shape generates a nonzero torque under the radiation pressure. We consider an incident ray with power $I$ and corresponding momentum $\vec{P}_{I}$ which falls on a surface element $d s \hat{n}$ of the trapped body. A fraction of the incident ray gets refracted and the momentum transfer gives rise to the gradient force that holds the cell at the point of focus. The remaining part gets reflected and gives rise to the scattering force. For objects that are symmetric about the optic axis, the radial and azimuthal components of the resultant force become zero. For the deformed erythrocytes (like the shape shown in figure 4), if they do not have any mirror symmetry about the optic axis, the azimuthal component of the scattering force does not become zero and hence produces a torque along the optic axis (Z-axis). In figure 4 , the change in momentum $\Delta \vec{P}$ produces an unbalanced and off-centered force which in turn causes a torque $\tau$, where

$$
\tau \propto \vec{r} \times \Delta \vec{P} .
$$

As $\Delta P \propto P_{I}$ and $P_{I} \propto I$, the torque therefore increases linearly with the incident power $I$. The torque also increases with increasing asymmetry as the distribution of $\vec{r}$ in eq. (2) is a measure of the asymmetry of this model shape (figure 4). Thus the speed or rotation has a linear relationship with the laser power for a fixed asymmetry and when the asymmetry is more, the rotational speed is higher at the same laser power. We have also observed the rotations of laser-trapped asymmetric nanorods that conform to the same rotation mechanism [12].

This rotation mechanism explains our observations completely. As the RBCs in isotonic and hypotonic osmolar conditions do not have enough asymmetry, they do not experience any torque under the optical trap. The RBCs show rotation only when they are in hypertonic buffer solution and bear shape asymmetry. The presence of bivalent ions makes the cell membrane stiff and the cells deform less, resulting in a slower rotation. When, because of higher concentration of bivalent ions the deformation of the cells are not sufficient to produce enough torque to 


\section{Manas Khan, Samarendra K Mohanty and A K Sood}

overcome the fluid drag, they do not rotate at all. Since the magnitude of the torque is independent of the state of polarization of the trapping beam, the observations are expected to be the same if a circularly polarized trapping beam is used instead of the linearly polarized beam.

\section{Conclusions}

We conclude that the deformation of the red blood cells in the hypertonic solution causes their rotation in the optical trap, the rotation being independent of the state of polarization of the trapping beam. Addition of bivalent ions makes the cell membrane stiffer resulting in a slower rotation. This RBC rotor can be used in many biology-related experiments where the $\mathrm{pH}$ and osmolarity of the medium are controlled. These can be used in microfluidic devices and to study micro-rheology.

\section{Acknowledgement}

AKS thanks the Department of Science and Technology, India, for financial support.

\section{References}

[1] A Ashkin, J M Dziedzic, J E Bjorkholm and S Chu, Opt. Lett. 11, 288 (1986)

[2] K Schutze, G Posl and G Lahr, Cell. Mol. Biol. 44, 735 (1998)

[3] M Zahn and S Seeger, Cell. Mol. Biol. 44, 747 (1998)

[4] A Clement-Sengewald, K Schutze, A Ashkin, G A Palma, G Kerlen and G Brem, J. Assist. Reprod. Genet. 13, 259 (1996)

[5] M Zahn, J Renken and S Seeger, FEBS Lett. 443, 337 (1999)

[6] C Bustamante, Z Bryant and S B Smith, Nature 421, 423 (2003)

[7] J A Dharmadhikari, S Roy, A K Dharmadhikari, S Sharma and D Mathur, Opt. Exp. 12, 1179 (2004)

[8] S K Mohanty, A Uppal and P K Gupta, Biotechnol. Lett. 26, 971 (2004)

[9] A Ghosh, S Sinha, J A Dharmadhikari, S Roy, A K Dharmadhikari, J Samuel, S Sharma and D Mathur, arXiv:physics/0501099 v1, 19 Jan. 2005

[10] S K Mohanty, K S Mohanty and P K Gupta, Opt. Exp. 13, 4745 (2005)

[11] J A Dharmadhikari, S Roy, A K Dharmadhikari, S Sharma and D Mathur, Appl. Phys. Lett. 85, 6048 (2004)

[12] M Khan, A K Sood, F L Deepak and C N R Rao, Nanomotors using asymmetric nanorods in optical trap (submitted)

[13] P Galajda and P Ormos, Opt. Exp. 11, 446 (2003)

[14] M Capitanio, G Romano, R Ballerini, M Giuntini and F S Pavone, Rev. Sci. Instrum. 73, 1687 (2002)

[15] E Evans and Y C Fung, Microvasc. Res. 4, 335 (1972)

[16] M Mela and S Eskelinen, Acta Physiol. Scand. 122, 515 (1984) 